\title{
Desarrollo metacognitivo en los docentes en educación: revisión sistemática
}

Metacognitive development in teachers in education: systematic review

Desenvolvimento metacognitivo em professores na educação: revisão sistemática

\section{ARTÍCULO GENERAL}

Karina Micaela Panta Carranza

pantakarina01@gmail.com

https://orcid.org/0000-0001-7231-3610

Investigador Independiente,

Lima - Perú
Jury Yesenia Aquino Trujillo

juryyeseniaaquinotrujillo@gmail.com

https://orcid.org/0000-0003-1662-6406

Investigador Independiente,

Lima - Perú
Jesús María Sosa Agurto

jsosaagurto@gmail.com https://orcid.org/0000-0001-8409-8946

Investigador Independiente, Lima - Perú

Recibido 02 de Diciembre 2021 | Arbitrado y receptado 14 de Marzo 2022 | Publicado en 02 Junio 2022

\section{Resumen}

La educación ha ido avanzando de acuerdo a las ciencias como la Pedagogía y Psicología, provocando que la función docente vaya cambiando, dejó de ser el único proveedor de información y el estudiante un pasivo receptor, restándole la posibilidad de refutar, opinar sobre el conocimiento brindado, crear y aplicarlo en su entorno.

De acuerdo a nuevas teorías, estudios en beneficio y avances de la educación, se ha ido incluyendo con mayor arraigo los procesos cognitivos, metacognitivos, empíricos, nuevas metodologías en la que se produce un gran cambio en el rol docente, convirtiéndose en facilitador, orientador del estudiante para que él pueda gestionar su conocimiento y aprendizaje.

\section{Abstract}

Education has been advancing according to the sciences such as Pedagogy and Psychology, causing the teaching function to change, it ceased to be the only provider of information and the student a passive receiver, reducing the possibility of refuting, giving an opinion on the knowledge provided. , create and apply it in your environment.

According to new theories, beneficial studies and advances in education, cognitive, metacognitive, empirical processes have been included with greater roots, new methodologies in which there is a great change in the teaching role, becoming a facilitator, counselor of the student so that he can manage his knowledge and learning.

\section{Resumo}

A educação vem avançando de acordo com as ciências como a Pedagogia e a Psicologia, fazendo com que a função docente mude, deixando de ser o único provedor de informações e o aluno um receptor passivo, diminuindo a possibilidade de refutar, opinar sobre o conhecimento fornecido ., crie e aplique em seu ambiente.

De acordo com novas teorias, estudos benéficos e avanços na educação, processos cognitivos, metacognitivos, empíricos foram incluídos com maiores raízes, novas metodologias nas quais há uma grande mudança no papel do professor, tornando-se um facilitador, conselheiro do aluno para que ele pode gerir o seu conhecimento e aprendizagem. 
En nuestro país, en esta época de cambios y nuevos retos, donde la presencialidad no se está dando, se debe continuar con la Educación, lograr aprendizajes significativos, independientemente del medio y forma de conexión, en la que se debe aprovechar hasta las necesidades, escases, dificultades para continuar aprendiendo y de manera reflexiva, crítica y creativa buscando nuevas soluciones, aprender a sobrellevarlas; en este entorno, se debe aprovechar el desarrollo metacognitivo, es decir enseñarles a los estudiantes aprender a aprender y pueda transferir sus aprendizajes en su vida cotidiana, tal como lo plantea Osses y Mora (2018) que es "necesario formar alumnos más conscientes y autónomos en sus aprendizajes, sin olvidar el aspecto motivacional y el contexto apropiado, en el desarrollo de las estrategias de aprendizaje" (p.196). Entonces, se debe abordar dos aspectos importantes como son la metacognición, docentes y la relación entre ellos.

En la actualidad ante las nuevas tendencias del conocimiento se está considerando la metacognición como un proceso activo, etimológicamente meta, proviene del prefijo griego que significa "más allá de" y cognición del latín cognoscere que significa conocer, es decir que es pensar sobre lo que se piensa, o como muchos autores consideran como aprender a aprender, permitiendo que la persona forme su autoconciencia y autorregule sus aprendizajes (Jaramillo \& Simbaña, 2014; Osses \& Mora, 2018). Uno de los pioneros en abordar la metacognición es Flavell, definiéndolo como conocimiento de nuestros propios procesos y productos cognitivos o lo que esté relacionado, posteriormente junto a Brown demuestran la efectividad en la comprensión lectora, donde los lectores exitosos eran los que tenían alto conocimiento cognitivo, esto ha conllevado a continuar investigando los beneficios en el proceso de enseñanza-aprendizaje (Valenzuela, 2018).

En este proceso de enseñanza y aprendizaje, es importante el docente, cuyo quehacer pedagógico debe considerar ser sociable, tener actitud de predisposición y reflexionar ante los cambios como percibir, pensar, sentir y comportarse ante un objeto o situación, debe desarrollar habilidades y competencias necesarias para el logro de aprendizajes de sus estudiantes (Londoño et al., 2017). Los docentes deben ser metacognitivos, asumir compromisos adaptando sus prácticas pedagógicas o quehacer educativo, sean eficaces en sus enseñanzas, estar "consciente de sus potencialidades y limitaciones, planificando, controlando y evaluando“(Osses \& Mora, 2018, p.

196). 
Valenzuela (2018) plantea en su artículo la necesidad de que el docente indague, conozca y se apropie de estrategias pedagógicas y esté consciente de cómo desarrollar la metacognición con los estudiantes, pero cuidando que este no se vuelva automatizado si no reflexivo. Gómez y Sandoval (2019) reafirma en su artículo que se debe alfabetizar metacognitivamente "como adquisición de conciencia, conocimiento y experiencia práctica acerca de una serie de habilidades cognitivas, metacognitivas y autorregulatorias fundamentales en la resolución de problemas, toma de decisiones y pensamiento crítico" (p.22). Entonces, al desarrollar e integrar los distintos aspectos metacognitivos el docente podrá orientar con autodirecciones, aprenderán a aprender de manera autónoma, reflexiva y crítica, por ende el logro de competencias, superándose la mecanización o superficialidad de la metacognición planteado en la actualidad como parte de la metodología para desarrollar las competencias del Currículo Nacional.

Como se puede percibir existe un bagaje de información sobre este tema; sin embargo no se aborda por novedad si no por necesidad, porque al cuestionarse y reflexionar permitirá que el docente reconozca lo que conoce, desconoce y necesita, tome conciencia, se apropie y desarrolle la metacognición para el logro de un aprendizaje significativo de los estudiantes, que a su vez sabrán cómo enfrentarse a otras situaciones dentro o fuera de la escuela, los aprendizajes ya no quedarán en el olvido o lo verán como inútil, porque desarrollaron adecuadamente habilidades metacognitivas por ende aprendizajes de por vida y aplicables en diversas situaciones (Gómez \& Sandoval, 2019).

Pero la Metacognición se está asumiendo de manera mecánica o irrelevante en la metodología, porque figura como parte de un formato estructurado al final o cierre de una sesión de aprendizaje, cuya aplicación se reduce preguntando al estudiante ¿qué aprendiste?, ¿cómo lo aprendiste? y ¿cómo lo vas a aplicar?

(MINEDU, 2016).

Obteniéndose como respuestas poco o nada trascendentales, inconsistentes, que conllevan al olvido o como un apunte más, en sus textos. Percibiéndose en el docente, desconocimiento de la importancia del proceso metacognitivo y su relación con la significancia de los aprendizajes por falta de identidad metacognitiva. Por tanto el objetivo del presente artículo será determinar las características metacognitiva de la práctica docente entorno a los conocimientos y conciencia. 


\section{Estado del arte}

Cárdenas et al. (2017) proponen mejorar el proceso de comprensión lectora en estudiantes del nivel primario utilizando estrategias metacognitivas, en sus conclusiones se puede destacar la importancia de transformar la manera de enseñar de un docente, en el que debe considerar una toma de conciencia, reflexionar sobre su quehacer pedagógico, ser un constante evaluador de lo que utiliza para conllevar a un aprendizaje, anticiparse a posibles situaciones ajenas a lo planteado, conocer el contexto, expectativas, características de los estudiantes para partir desde ellos y monitorear, evaluar y autoevaluar constantemente le proceso pedagógico para identificar dificultades, buscar cómo superarlas y lograr en el estudiante un aprendizaje significativo.

En otra de sus conclusiones y sustenta uno de los puntos a investigar, es la forma de cómo se debe abordar la evaluación, no como un instrumento final y sancionador, si no que sea formativo, que permita identificar los logros o dificultades del estudiante, ante ello orientarlo hasta que logre un aprendizaje significativo.

Continuando con las revisiones de acuerdo a Valenzuela (2018) en su artículo aborda como objetivo identificar el desarrollo y tendencias de las investigaciones relacionadas con la metacognición y su aplicación en dos procesos de lectura y escritura en un nivel académico superior, concluyendo que la metacognición ha estado relacionado con la lectura y escritura, obteniéndose resultados que contribuyen a la autorregulación, desarrollo de lecto-escritura y aspectos socio comunicativas, pero "no hay una presencia significativa de estudios que examinen la relación entre la metacognición y los procesos de alfabetización académica" (P.85).

Asimismo, propone que se defina, delimite componentes y se establezca una taxonomía en la aplicación de estrategias en educación superior. En consecuencia, nuevamente reafirma este autor la necesidad de que el docente indague, conozca y se apropie de estrategias pedagógicas y esté consciente de cómo desarrollar la metacognición con los estudiantes, pero cuidando que este no se vuelva automatizado si no reflexivo.

Gómez y Sandoval (2019) en su artículo sobre la alfabetización metacognitiva para el desarrollo de la comprensión auditiva del español como segunda lengua, sustentan la importancia y qué debemos realizar para desarrollar la metacognición, plantean 
“alfabetización metacognitiva como adquisición de conciencia, conocimiento y experiencia práctica acerca de una serie de habilidades cognitivas, metacognitivas y autorregulatorias fundamentales en la resolución de problemas, toma de decisiones y pensamiento crítico" (p.22). En consecuencia, concuerdo que se debe alfabetizar metacognitivamente, porque al desarrollar e integrar los distintos aspectos metacognitivos el docente podrá orientar con autodirecciones, aprenderán a aprender de manera autónoma, reflexiva y crítica, por ende el logro de competencias, superándose la mecanización o superficialidad de la metacognición planteado en la actualidad como parte de la metodología para desarrollar las competencias del Currículo Nacional.

\section{METODOLOGÍA}

El método empleado para el estudio, consistió en una revisión sistemática de artículos científicos indexados en bases de datos científicas con verificable rigor académico en ciencias psicológicas y ciencias de la educación; esta fueron:

Scopus, Scielo, Web of Science, Google Scholar; y Elsevier.

\section{Proceso de selección}

Los criterios para la elección de los artículos se basaron en: 1) participantes docentes o docentes en formación, 2) idioma español e inglés, 3) de nivel doctoral o magister, 4) fecha de búsqueda 2018 al 2020, 5) palabras claves en: títulos, descriptores, objetivos., 6) enfoque cualitativo, cuantitativo y mixto, y 7) palabras claves en español como Metacognición, Aprendizaje, docente, además se consideraron en inglés metacognitive literacy, teacher, metacognitive strategies. Los operadores lógicos que acompañaron en la ecuación de búsqueda fueron "y”, "or, "and"

El proceso de indagación fue efectuado en los meses de noviembre y diciembre del presente año, encontrando 14.300 resultados; con el posterior proceso de selección, eliminando coincidencias, grupo de estudio, acceso a la información; en total fueron analizados doce artículos académicos. Para la organización y almacenamiento se utilizó el gestor de bibliografías Zotero. 


\section{RESULTADOS}

De acuerdo al objetivo de determinar la importancia de desarrollar la metacognición en los docentes para lograr aprendizajes significativos. Para sustentar y atender a las distintas preguntas planteadas se procedió a una búsqueda en cinco Base de Datos:

\section{Criterio especifico de elegibilidad}

\begin{tabular}{|c|c|c|c|c|c|c|}
\hline Refinación & Scopus & $\begin{array}{l}\text { Web of } \\
\text { Science }\end{array}$ & Elsevier & $\begin{array}{l}\text { Google } \\
\text { Scholar }\end{array}$ & Scielo & \\
\hline Descriptores & \multicolumn{4}{|c|}{$\begin{array}{l}\text { Metacognition and teaching or Metacognition and } \\
\text { Teacher or Metacognitive devolpment and teacher or } \\
\text { Metacongitive skills and teacher or } \\
\text { Metacongitive thinking and teacher }\end{array}$} & $\begin{array}{l}\text { Metacognición } \\
\text { docente } \\
\text { Formación } \\
\text { docente y } \\
\text { metacognición }\end{array}$ & \\
\hline Periodo & $\begin{array}{l}2018- \\
2020\end{array}$ & $\begin{array}{l}2018- \\
2020\end{array}$ & $\begin{array}{l}2018- \\
2020\end{array}$ & $\begin{array}{l}2018- \\
2020\end{array}$ & $2018-2020$ & \\
\hline Áreas & Todas & Todas & Todas & Todas & Todas & \\
\hline Idioma & $\begin{array}{l}\text { Español e } \\
\text { inglés }\end{array}$ & $\begin{array}{l}\text { Español e } \\
\text { inglés }\end{array}$ & $\begin{array}{l}\text { Español e } \\
\text { inglés }\end{array}$ & $\begin{array}{l}\text { Español e } \\
\text { inglés }\end{array}$ & $\begin{array}{l}\text { Español } \\
\text { inglés }\end{array}$ & $\mathrm{e}$ \\
\hline $\begin{array}{l}\text { Tipo } \\
\text { de } \\
\text { documento }\end{array}$ & Artículo & Artículo & Artículo & Artículo & Artículo & \\
\hline $\begin{array}{r}\text { Grupo } \\
\text { de } \\
\text { estudio }\end{array}$ & Docentes & docentes en fo & aación & & & \\
\hline Nivel & $\begin{array}{l}\text { Magíster } \\
\text { y } \\
\text { Doctoral }\end{array}$ & $\begin{array}{l}\text { Magíster } \\
\text { y } \\
\text { Doctoral }\end{array}$ & $\begin{array}{l}\text { Magíster } \\
\text { y } \\
\text { Doctoral }\end{array}$ & $\begin{array}{l}\text { Magíster } \\
\text { y } \\
\text { Doctoral }\end{array}$ & $\begin{array}{l}\text { Magíster } \\
\text { Doctoral }\end{array}$ & $\mathrm{y}$ \\
\hline
\end{tabular}




\section{Proceso de análisis}

El registró de los datos se realizó por medio de una selección de características de los artículos académicos, con los siguientes campos: fuente, país, año, objetivos, metodología, conclusiones principales. 


\begin{tabular}{|c|c|c|c|}
\hline 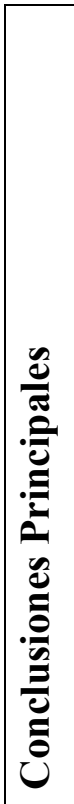 & 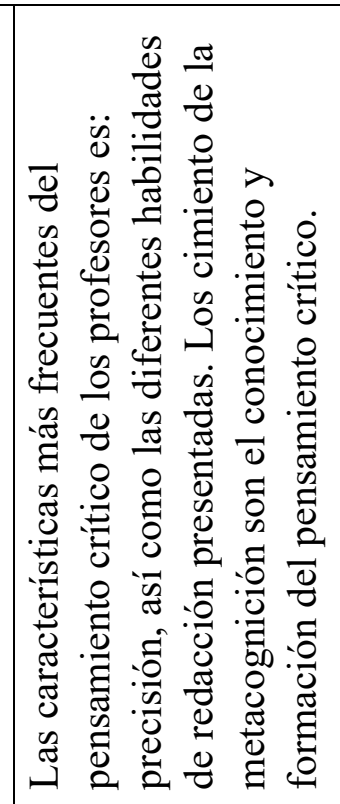 & 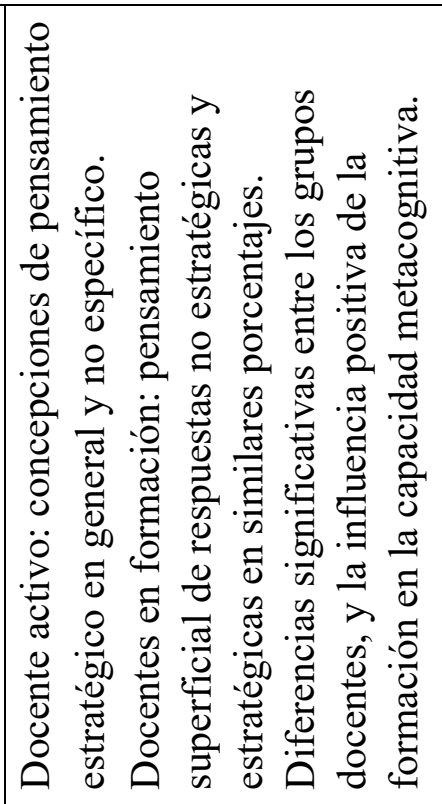 & 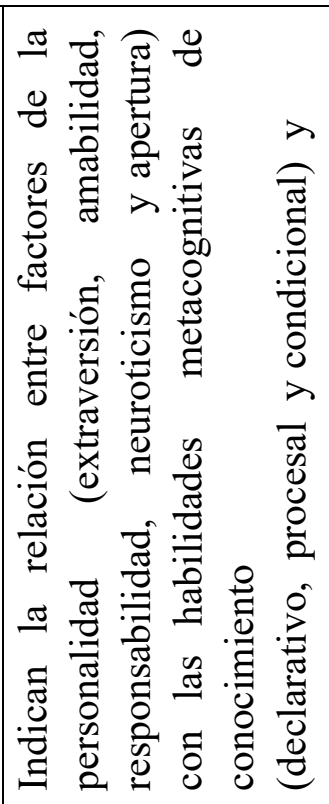 \\
\hline 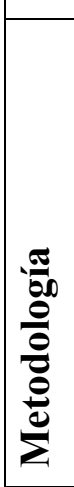 & 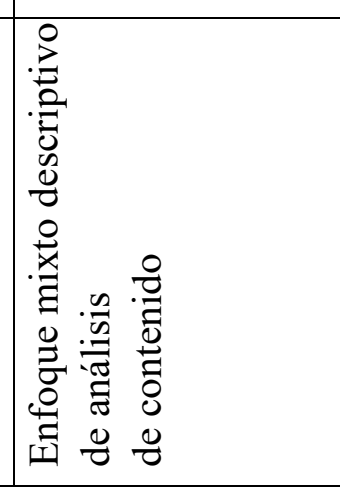 & 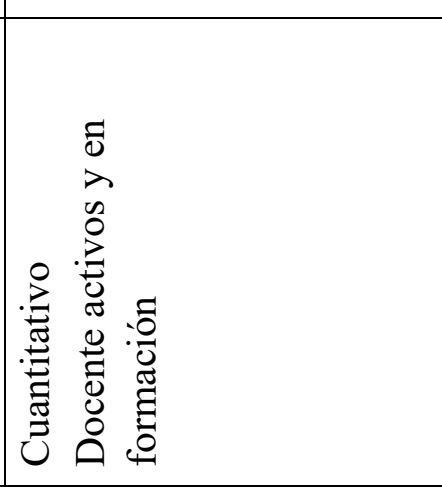 & 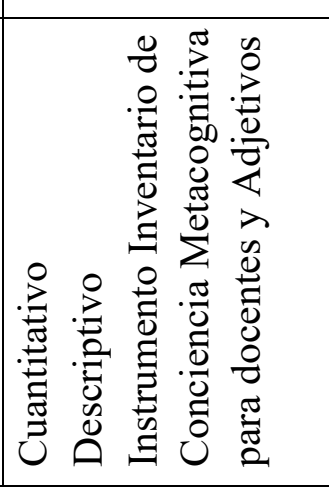 \\
\hline 晜 & 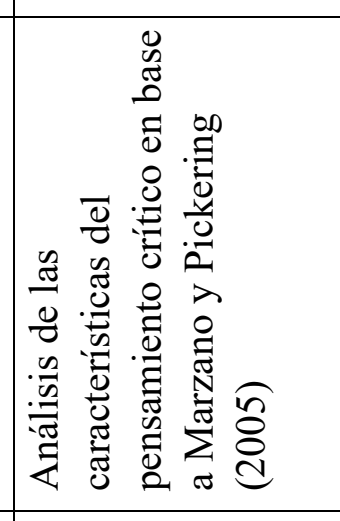 & 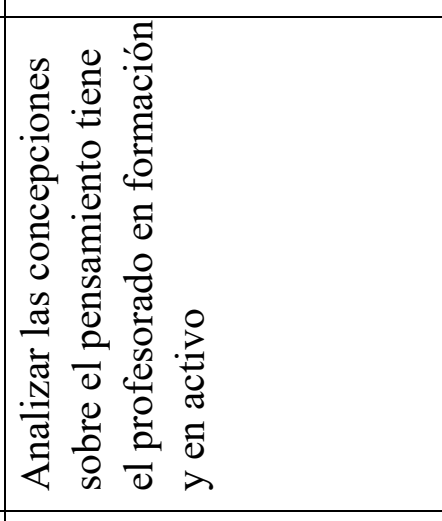 & 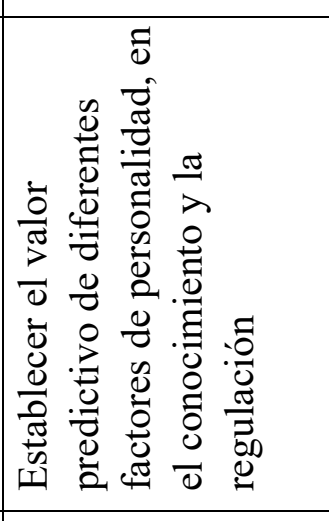 \\
\hline 施 & 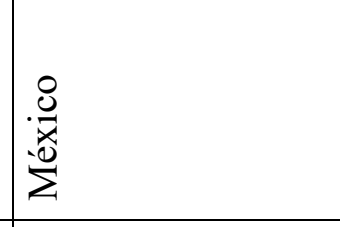 & 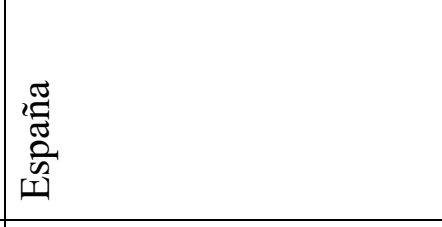 & \begin{tabular}{|l}
$\frac{\pi}{0}$ \\
$\frac{1}{0}$ \\
$\frac{0}{8}$ \\
\end{tabular} \\
\hline 导 & $\stackrel{\infty}{\stackrel{\sim}{\sim}}$ & ڤे̀े & ָัे \\
\hline 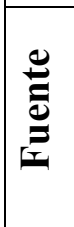 & 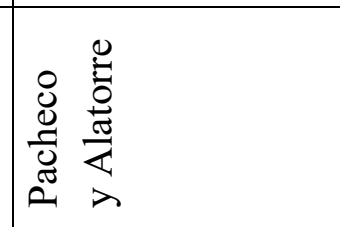 & 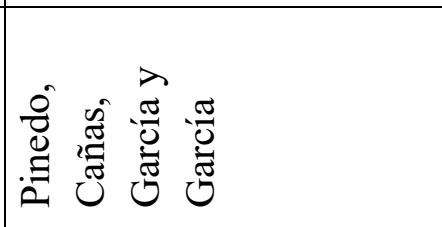 & 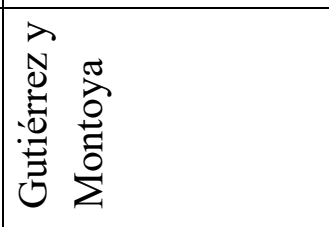 \\
\hline
\end{tabular}




\begin{tabular}{|c|c|c|}
\hline 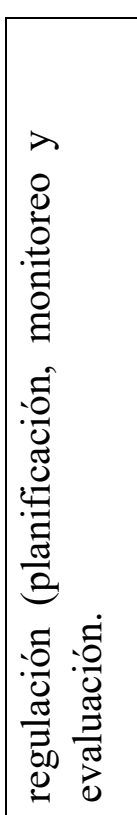 & 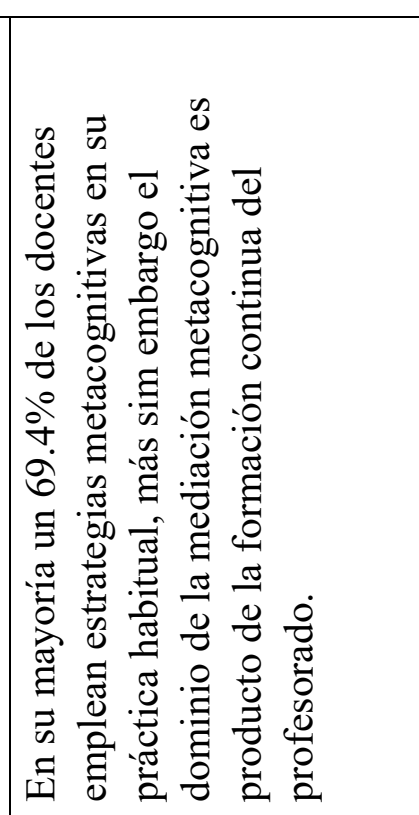 & 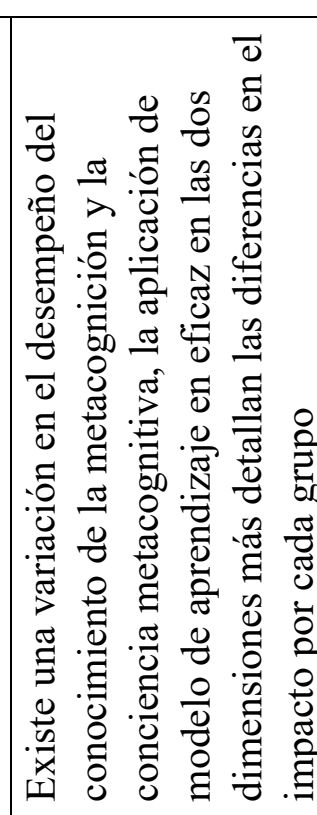 \\
\hline 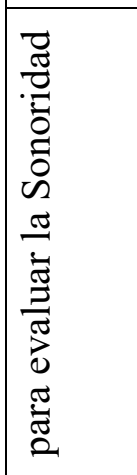 & 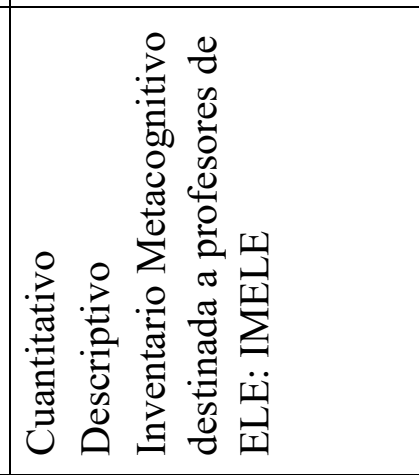 & 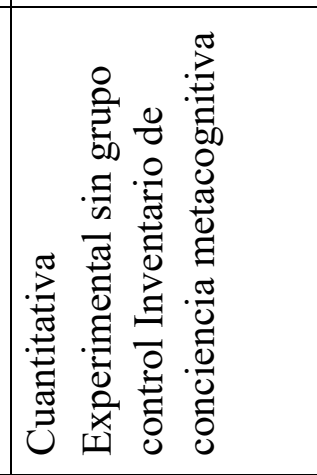 \\
\hline 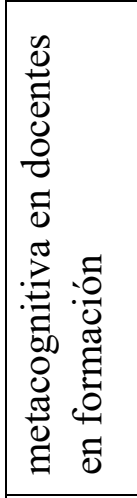 & 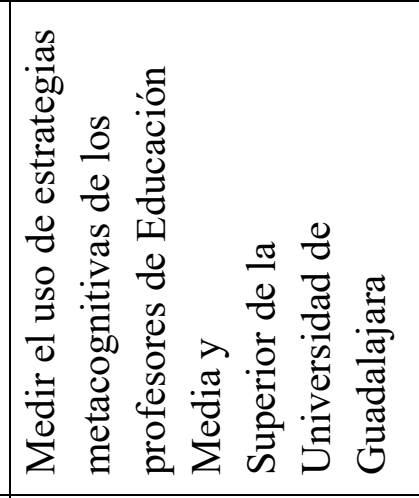 & 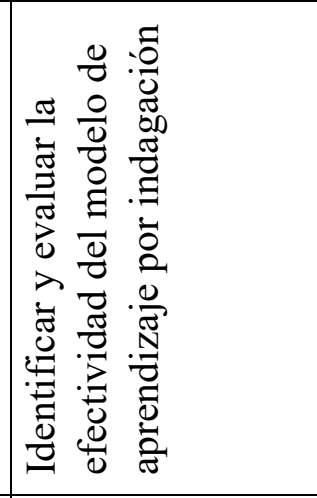 \\
\hline & 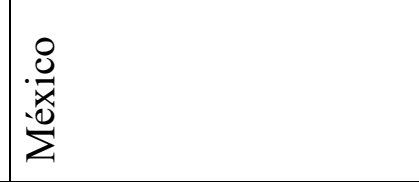 & 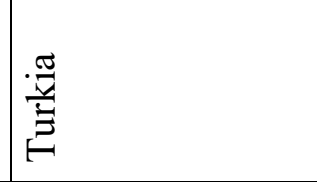 \\
\hline & 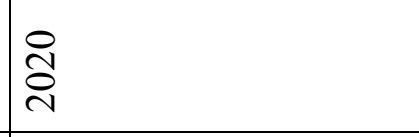 & 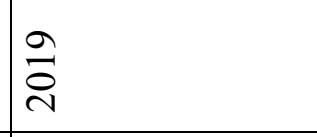 \\
\hline & 兽: & 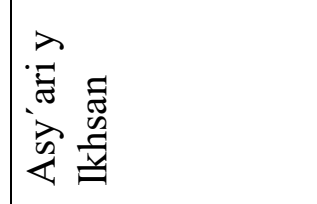 \\
\hline
\end{tabular}




\begin{tabular}{|c|c|}
\hline 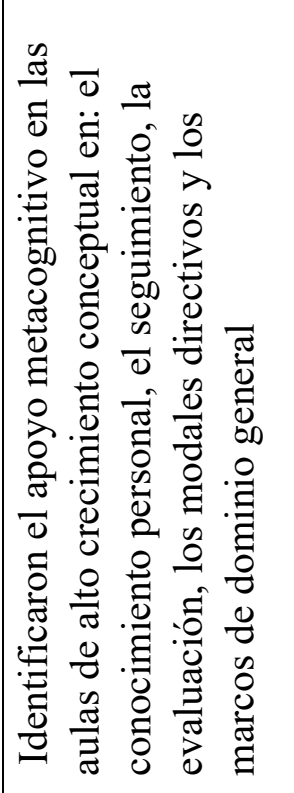 & 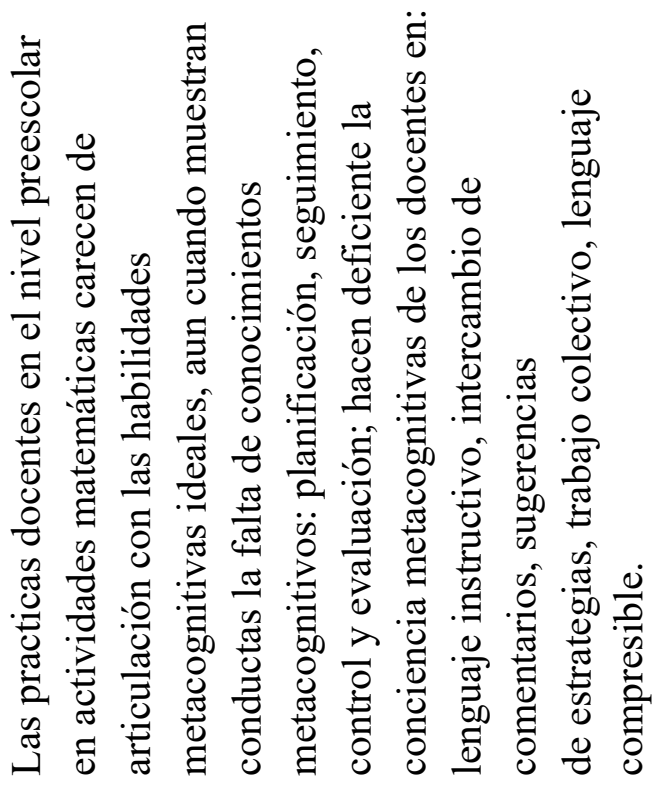 \\
\hline 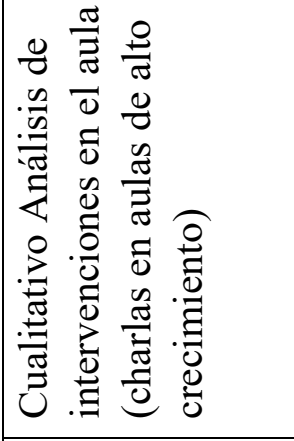 & 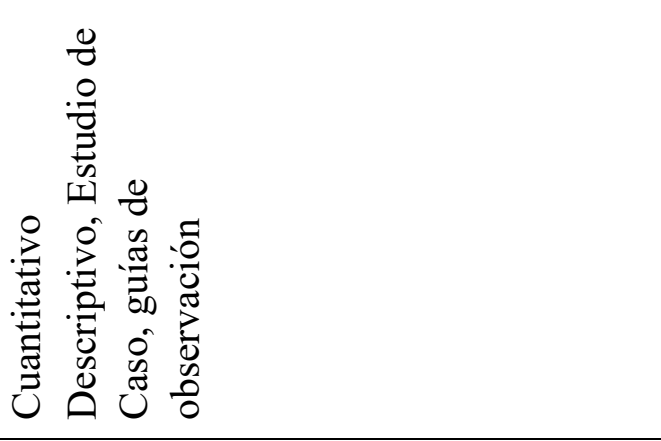 \\
\hline 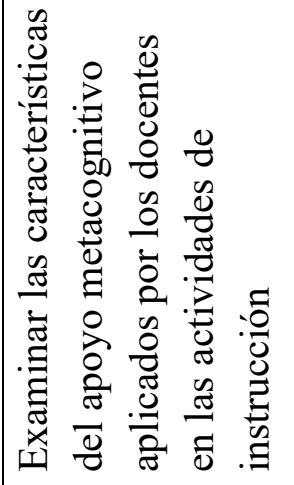 & 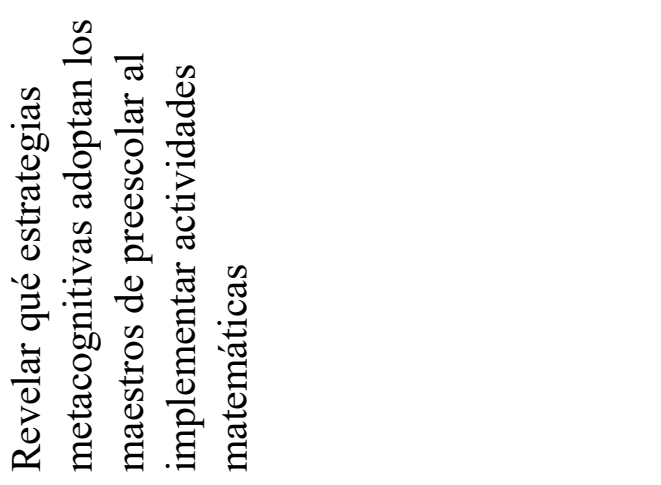 \\
\hline$\underset{⿱ 乛 龰}{\overleftrightarrow{S}}$ & : \\
\hline$\stackrel{\partial}{\stackrel{\partial}{\nu}}$ & $\stackrel{\stackrel{\curvearrowright}{\circ}}{\stackrel{\sim}{ }}$ \\
\hline 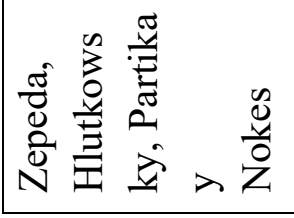 & 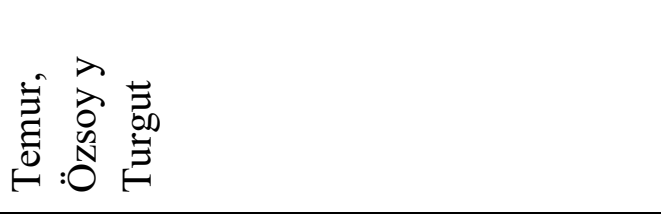 \\
\hline
\end{tabular}




\begin{tabular}{|c|c|}
\hline 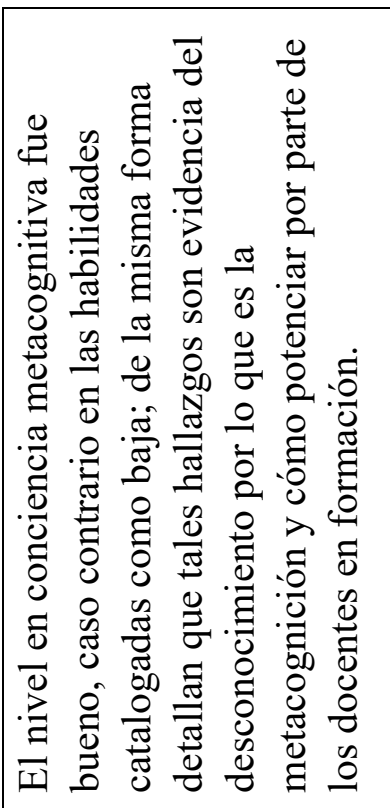 & 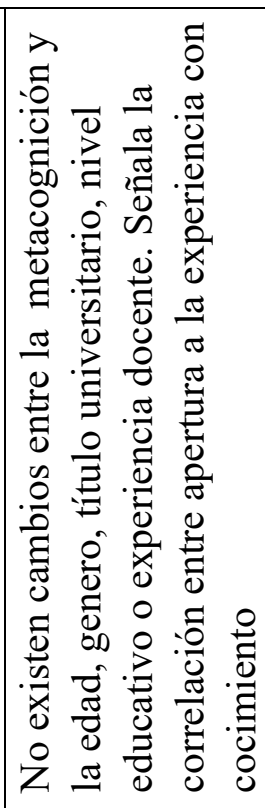 \\
\hline 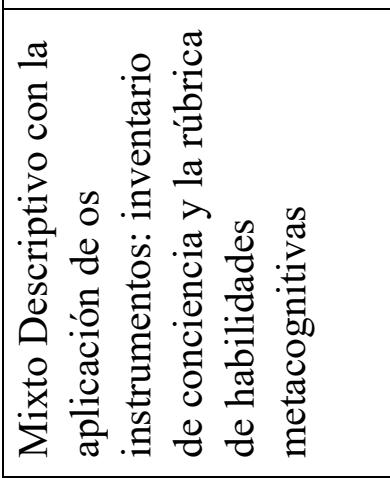 & 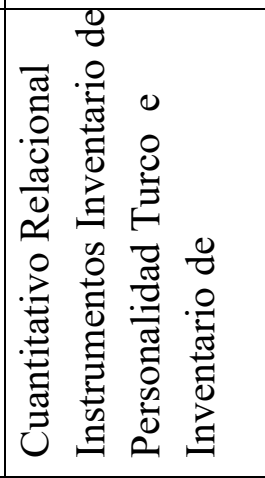 \\
\hline 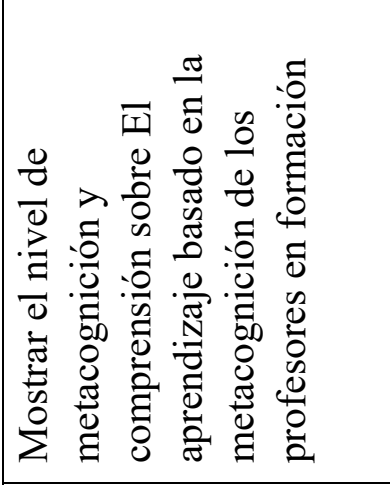 & 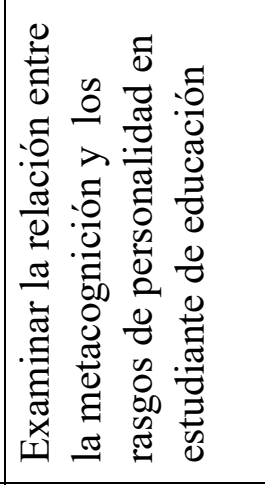 \\
\hline $\begin{array}{l}. \frac{\pi}{\pi} \\
\frac{\mathscr{D}}{0} \\
\frac{0}{0} \\
\Xi\end{array}$ & $\underset{\Xi}{\stackrel{0}{\Xi}}$ \\
\hline$\stackrel{\partial}{\stackrel{\partial}{\nu}}$ & 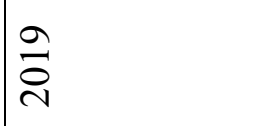 \\
\hline 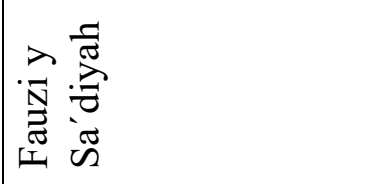 & 凉 \\
\hline
\end{tabular}

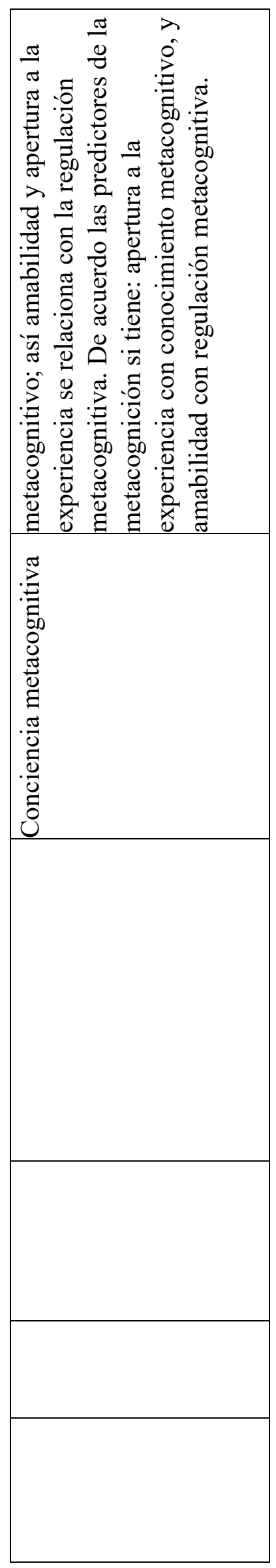




\begin{tabular}{|c|c|}
\hline 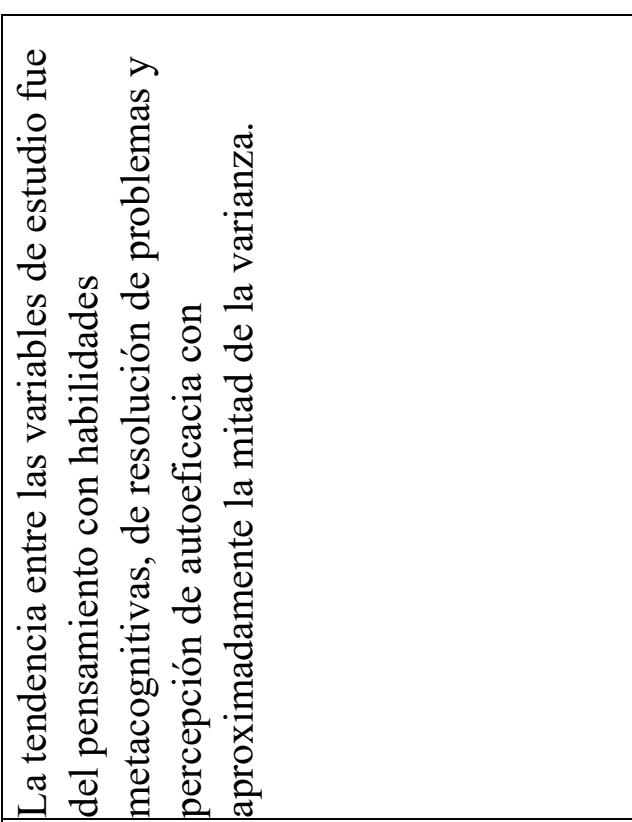 & 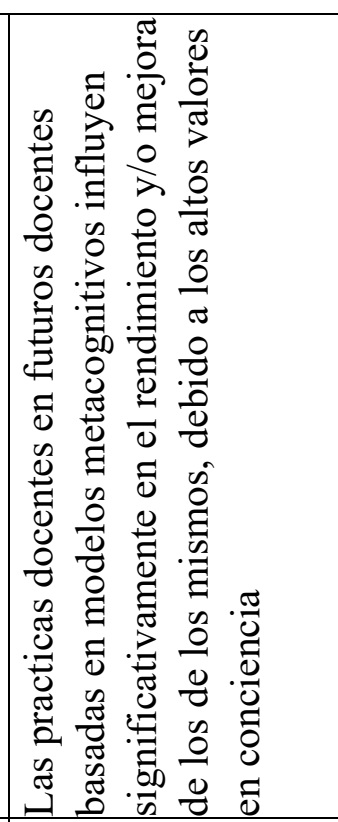 \\
\hline 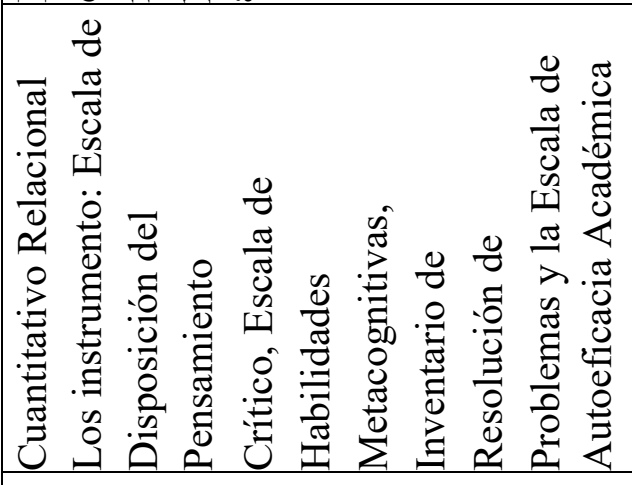 & 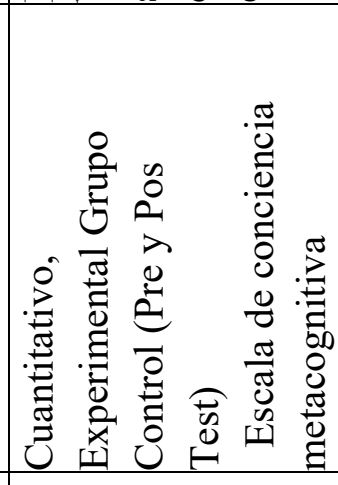 \\
\hline 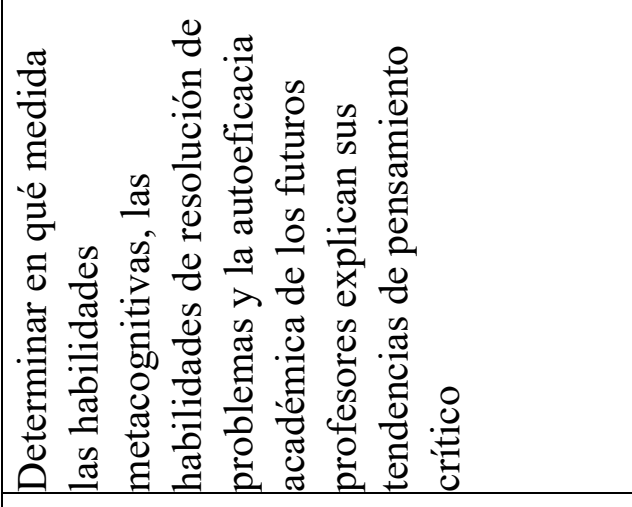 & 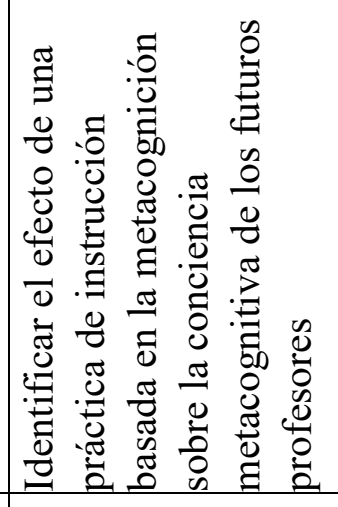 \\
\hline 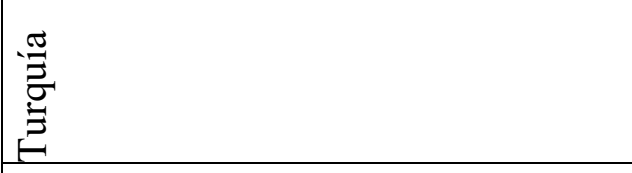 & 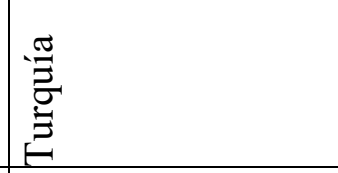 \\
\hline$\stackrel{\vec{\partial}}{\vec{\nu}}$ & $\frac{a}{\partial}$ \\
\hline 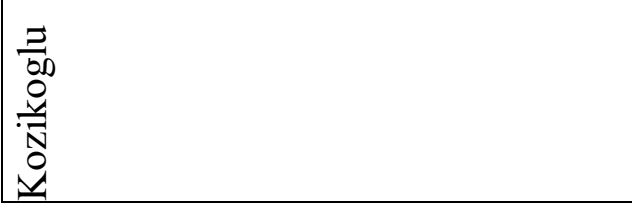 & 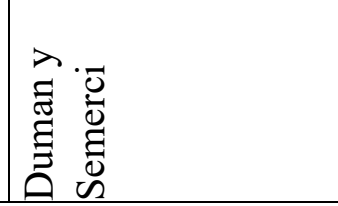 \\
\hline
\end{tabular}

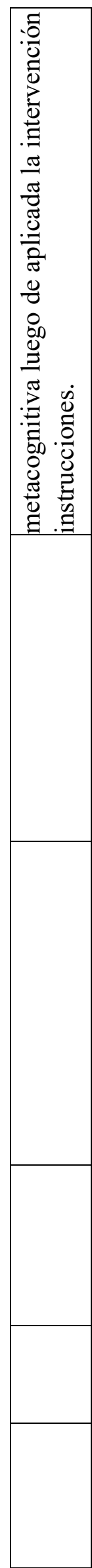

急焉 


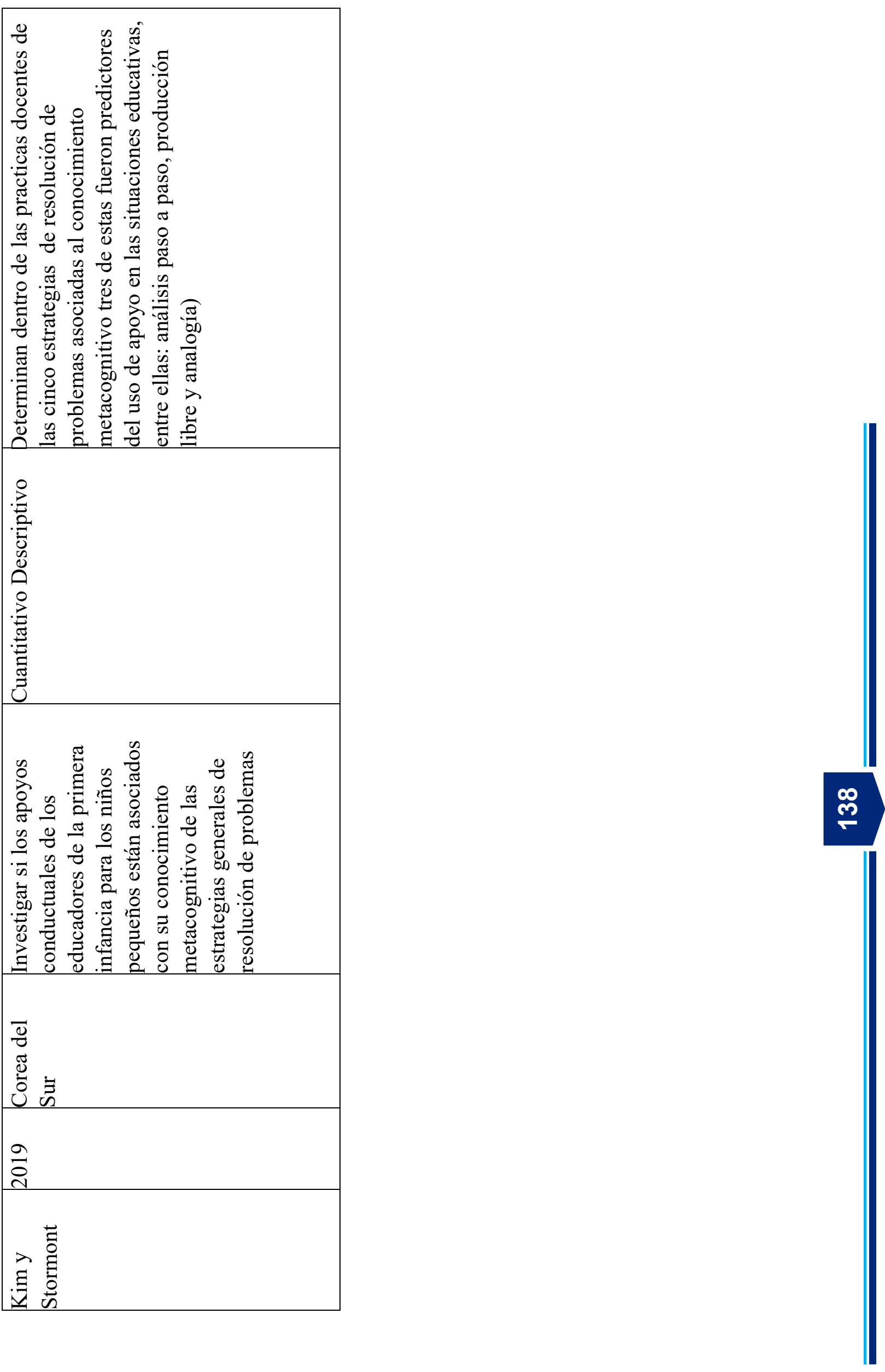




\section{Discusión}

De acuerdo a la revisión documental en las fuentes seleccionadas atendiendo al objetivo de analizar el desarrollo en conocimientos y conciencia metacognitivas en los docentes y en los docentes en formación, se presentan un conjunto de predictores de la práctica profesional en diéntenles áreas como la redacción científica, la resolución de problemas, la toma de daciones, las habilidades mentales en diversas áreas de conocimiento.

Si bien es cierto, que las condiciones laborales en cada país presentan diferentes aristas a favor o en contra del aspecto de divergencia de pensamientos, es necesario incluir un condición sin el cual no, es permisible la adopción de la metacognición en las prácticas educativas, como lo es la formación continua; como detalla Pinedo, Cañas, García y García (2019) y Pacheco y Alatorre (2018) dónde el nivel en conciencia metacognitiva así como en sus habilidades presenta diferencias significativas entre un docente con más de 10 años de servicio a un estudiante en los últimos semestres de escolaridad.

Más sin embargo, el condicionante de formación contenía se encuentra afectado por las dimensiones de la personalidad, señalado por Ozturk (2019) y Temur, Özsoy y Turgut (2019) siendo diferencias por la apertura a la experiencia y la amabilidad; es evidente que todas ellas hablando en términos de motivación y autoeficacia permiten al docente crear los motivos para la búsqueda de la perfección profesional, incluyendo el termino de mediación metacognitiva (Galindo, Gutiérrez, Ruíz y Martínez, 2020), llegando a señalar a equiparar los aspectos de formación y mediación metacognitiva en la tan esperada optimalización del pensamiento.

Si se habla en términos de gestor de conocimientos o promotor de ellos; el docente en el aula de clase es el líder del grupo y por tanto, esa posición, no es tomar el visión autoritaria común y replicada de muchos ambientes educativos, no; se estaría hablando del papel visionario y resonante de la práctica profesional docente; y son las situaciones de la medición metacognitiva que trasciende tales demandas del sistema educativo a nivel mundial; si en el aula el líder es incapaz de cambiar su pensamiento, de entrar en la rutina, de incitar la individualidad más que la colectividad, de ser incongruente con sus gestos y/o palabras con lo que en verdad es el propósito de la educación inclusiva y significativa; el cambio transformacional del mismo repercutirá en sus estudiantes. 


\section{Conclusiones}

Considerando la importancia de cambiar el sujeto mediador de conocimiento en aras de optimizar en ambiente educativo, las situaciones para conocer y/o identificar los componentes de las habilidades y los conocimientos metacognitivas para una educación significativa, seria cambiar el pensamiento del mediador y del sujeto a mediar, cambiando el entorno desde las concepciones previas hacia trasformadores del pensamiento, varios son los factores predictivos y condicionantes de la conciencia y conocimiento metacognitivo: personalidad, pensamiento crítico, autoeficacia, resolución de problemas, toma de decisiones; pero es desde las formas de cómo aplica sus estrategias personales en sus estudiantes que el cambio de pensamiento lograr prevalecer en los diferentes ambientes, modalidades y entornos:

En resolución de problemas: análisis pasó a paso, producción libre y analogía. (Kim y Stormont, 2019)

En conciencia metacognitiva: lenguaje instructivo y evaluativo, ambiente motivado al intercambio de ideas, sugerencias de estrategias por los estudiantes, protagonismo en las actividades colectivas, ambiente socializador. (Temur, Özsoy y Turgut, 2019)

En el pensamiento crítico: precisión, búsqueda, análisis, organizadores visuales. (Pacheco y Alatorre, 2018)

\section{Referencias bibliográficas}

Asy'ari, M., Ikhsan, M., \& Muhali. (2019). The Effectiveness of Inquiry Learning Model in Improving Prospective Teachers' Metacognition Knowledge and Metacognition Awareness. International Journal of Instruction, 12(2), 455470.

Blume, A. G., \& Montoya-Londoño, D. (2020). Relación entre factores de personalidad y metacognición en una muestra de estudiantes del último semestre de formación de programas de licenciatura en Educación en Colombia. Educación y Humanismo, 22(39), Article 39. https://doi.org/10.17081/eduhum.22.39.4048

Cárdenas, L., Parra, L., Pérez, A., \& Rincón, E. (2017). Las estrategias metacognitivas como herramientas transformadoras de las prácticas de aula para fortalecer la 
comprensión lectora de los estudiantes de básica primaria de la I.E.D Gonzalo Jiménez de Quesada del municipio de Suesca Cundinamarca] [Universidad de la Sabana].

https://intellectum.unisabana.edu.co/bitstream/handle/10818/33266/trabajo grado. pdf? sequence $=5 \&$ is Allowed $=\mathrm{y}$

Duman, B., \& Semerci, Ç. (2019). The Effect of a Metacognition-Based Instructional Practice on the Metacognitive Awareness of the Prospective Teachers. Universal Journal of Educational Research, 7(3), 720-728.

Fauzi, A., \& Sa'diyah, W. (2019, enero 1). The Metacognition of Pre-Service Biology Teachers: Awareness, Skills, Understanding, and Practices. Advances in Social Science, Education and Humanities Research, 349. https://doi.org/10.2991/iccd$\underline{19.2019 .8}$

Gómez, L., \& Sandoval, M. (2019). Alfabetización metacognitiva para el desarrollo de la comprensión auditiva en segundas lenguas: Fundamentos para la didáctica de ELE. Doblele. Revista de Lengua y Literatura, 5(0), $19 \quad$ - 46. https://doi.org/10.5565/rev/doblele.56

González, R. M. G., Galindo, P. A. G., Aguirre, E. I. R., \& Cruz, N. L. M. de la. (2020). Utilización de la mediación metacognitiva por los profesores de educación media y superior. Revista Electrónica Sobre Cuerpos Académicos y Grupos de Investigación, 7(14), 68-86.

Jaramillo Naranjo, L. M., \& Simbaña Gallardo, V. P. (2014). La metacognición y su aplicación en herramientas virtuales desde la práctica docente. Sophía, 16(16), 300313. https://doi.org/10.17163/soph.n16.2014.13

Kim, Y. H., \& Stormont, M. (2020). Early Childhood Teachers' Ratings of Metacognitive Knowledge and Behavioral Support Strategy Use in Classrooms. Early Childhood Education Journal, 48(2), 181-188. https://doi.org/10.1007/s10643-019-00991-z

Kozikoğlu, I. (2019). Investigating Critical Thinking in Prospective Teachers: Metacognitive Skills, Problem Solving Skills and Academic Self-Efficacy. Journal of Social Studies Education Research, 10(2), 111-130. 
Kyriakides, L., Anthimou, M., \& Panayiotou, A. (2020). Searching for the impact of teacher behavior on promoting students' cognitive and metacognitive skills. Studies in Educational Evaluation, 100810. https://doi.org/10.1016/j.stueduc.2019.100810

Londoño, O., Calderón, L., Lucumí, P., \& González, M. (2017). Experiencia innovadora de la estructuración ontológica del conocimiento con docentes y estudiantes. Praxis \& Saber, 8(16), 83. https://doi.org/10.19053/22160159.v8.n16.2017.6169

MINEDU. (2016). Sesiones de Aprendizaje versión 2016. http://www.minedu.gob.pe/rutasdelaprendizaje/sesiones2016/pdf/secundaria/unida d03/comunicacion/quint ogr ado/com-u3-5grado-sesion12.pdf

Osses, S., \& Mora, S. (2018). Metacognición: Un camino para aprender a aprender. Estudios Pedagógicos $\quad$ ～34(1), 187-197. https://doi.org/https://doi.org/10.4067/S0718-07052008000100011

Ozturk, N. (2020). Analysis of Teachers' Metacognition and Personality. PSYCHOLOGY AND EDUCATION, 57(1), 40-44.

Pacheco-Cortés, A. M., \& Alatorre-Rojo, E. P. (2018). La metacognición en la profesionalización docente: El pensamiento crítico en un entorno mixto.

Revista de Educación a Distancia (RED), 56, Article 56. https://revistas.um.es/red/article/view/321651

Pinedo González, R., Universidad de Valladolid, Cañas Encinas, M., Universidad de Valladolid, García Martín, N., Universidad de Valladolid, García González, N., \& EOEP Alcobendas. (2019). Capacidad metacognitiva en docentes y futuros docentes deenseñanza no universitaria. Revista de Psicología y Educación - Journal of Psychology and Education, 14(1), 74. https://doi.org/10.23923/rpye2019.01.173

Temur, Ö. D., Özsoy, G., \& Turgut, S. (2019). Metacognitive instructional behaviours of preschool teachers in mathematical activities. ZDM, 51(4), 655-666. https://doi.org/10.1007/s11858-019-01069-1

Valenzuela, Á. (2018). La metacognición en los procesos de lectura y escritura académica: ¿qué nos dice la literatura? (Vol. 46, Issue 1). https://doi.org/http://dx.doi.org/10.25100/lenguaje.v46i1.6197 
Zepeda, C. D., Hlutkowsky, C. O., Partika, A. C., \& Nokes-Malach, T. J. (2019). Identifying teachers' supports of metacognition through classroom talk and its relation to growth in conceptual learning. Journal of Educational Psychology, 111(3), 522-541. https://doi.org/10.1037/edu0000300 